\title{
Comparison of fatigue crack initiation behavior in different microstructures of TC21 titanium alloy
}

\author{
Changsheng Tan ${ }^{1}$, Qiaoyan Sun ${ }^{1 *}$, Lin Xiao ${ }^{1}$, Yongqing Zhao ${ }^{2}$, Jun Sun ${ }^{1}$ \\ 1. State Key Laboratory for Mechanical Behavior of Materials, Xi'an Jiaotong University, Xi'an, Shaanxi, 710049, P.R. \\ China \\ 2. Northwest Research Institute of Nonferrous Metals, Xi'an, Shaanxi, 710016, P.R. China \\ *Corresponding author. Tel.: +8629 82668614; fax: +862982663453. \\ E-mail address: qysun@mail.xjtu.edu.cn (Qiaoyan Sun).
}

\begin{abstract}
Cyclic heterogeneous deformation, slip characteristics and crack nucleation with different microstructures, such as bimodal microstructure (BM) and fine lamellar microstructure (FLM) in TC21 alloy (Ti-6Al-2Sn-2Zr-3Mo-1Cr-2Nb-0.1Si), were systematically investigated and analyzed during high cycle fatigue at room temperature. The results demonstrated that the FLM microstructure possesses higher high-cycle fatigue strength than those of the BM one. For $\mathrm{BM}$, the heterogeneous plastic deformation existed within the different large primary $\alpha$ phase, such as equiaxed primary $\alpha$ and primary $\alpha$ lath. The cracks at interfaces and slip bands easily coalesce with each other to form large cracks in BM. However, the $\alpha$ laths with similar morphology and size (nanosize) distributed uniformly in FLM and could relatively deform homogeneously in micro-region, which delayed the initiation of the fatigue crack. Based on the electron-backscattered diffraction (EBSD) analysis, it found that the strain was nonuniformly distributed in BM, however, it is relatively homogeneous in FLM. Moreover, lots of straight cracks are parallel and along single intrusions within the $\beta$ grain which delays the coalescence of cracks.
\end{abstract}

\section{Introduction}

Due to the high strength, high operating temperature, and corrosion resistance, titanium alloys have been applied widely to aerospace industries [1-3]. Based on the constitution of phase, the titanium alloy is generally classified into three groups: near $\alpha, \alpha+\beta$, and $\beta$ titanium alloys. TC21 titanium alloy is a new $\alpha+\beta$ structural material with high strength and fracture toughness and has been successfully developed and applied in aircraft structures [4-5]. However, during those applications, the aircraft structure parts are mainly subjected to cyclic loading, especially for the high cycle fatigue loading [6]. It has been reported that fatigue failure is one of the major problems for the application of materials in transportation, manufacturing, medical devices and electronic industries [7].

It has been considered that fatigue life is determined by crack initiation because major of the fatigue life is spent on this region during the high cycle fatigue [8-9]. The crack initiation and coalescence in titanium alloy can be varied significantly by the microstructural parameters, such as phase dimensions, volume fraction, and morphology [10-11]. Many researchers have systematically investigated the influence of microstructures on the fatigue crack initiation behavior in titanium alloys [11-16]. It is found that the fatigue strength decreases in the order of bimodal microstructure, lamellar microstructure and equiaxed microstructure in Ti-6Al-4V alloy [16]. Huang et al [13-15] found that some small-scale heterogeneous microstructure regions (SHMR) in $\beta$ grain interiors and the grain boundary (GB) $\alpha$ layer promote the crack initiation during fatigue loading in LM than BM which resulted in a lower fatigue strength for former one. While the crack initiation site depends on amount and size of primary $\alpha$ phase in bimodal microstructures, it can initiate either on the primary $\alpha / \beta$ transformation matrix or within the primary $\alpha$ phase [17]. Therefore, it is essential to reveal the relationship between micro-mechanism and fatigue life in titanium alloys.

During high cycle fatigue, the applied stress amplitude is often below the yield strength and still in the elastic region. However, it has been reported that it is not entirely elastic and the micro-plastic deformation occurs below the yield strength [18-20]. With further loading, localized plastic deformation was observed to initiate at individual grains with several preferred orientations which may result in crack formation [21]. This heterogeneous deformation within micro-regions 
can lead to a fatal failure after high cycles [19, 22-23]. However, little information is available in the literature $[10-11,25]$ about the strain distribution and heterogeneous deformation with different microstructures during high cycle fatigue in TC21 titanium alloy.

Thus, in present work, the cyclic heterogeneous deformation, slip characteristics, crack nucleation and coalescence were systematically analyzed. Subsequently, the slip irreversibility and accumulative irreversible strain in BM and LM were calculated to explore the cyclic heterogeneous and localized deformation.

\section{Material and experimental procedure}

TC21 titanium alloy was produced by the Northwest Institute for Nonferrous Metal Research in China. The cylinder samples with $15 \mathrm{~mm}$ in diameter, $80 \mathrm{~mm}$ in height were obtained from the as-received TC21 disc. The heat treatments for bimodal microstructure and fine lamellar microstructure are listed in table 1.

Table 1. Heat treatments for the two microstructures

\begin{tabular}{|c|c|c|c|c|}
\hline Structures & $\begin{array}{c}\text { Temperature } \\
\left({ }^{\circ} \mathrm{C}\right)\end{array}$ & $\begin{array}{c}\text { Time } \\
(\mathrm{h})\end{array}$ & $\begin{array}{c}\text { Temperature } \\
\left({ }^{\circ} \mathrm{C}\right)\end{array}$ & $\begin{array}{c}\text { Time } \\
(\mathrm{h})\end{array}$ \\
\hline $\mathrm{BM}$ & 900 & 1 & 600 & 4 \\
\hline FLM & 970 & 1 & 600 & 4 \\
\hline
\end{tabular}

* Both were air cooled

Tensile specimens with a diameter of $6 \mathrm{~mm}$ and a gage length of $30 \mathrm{~mm}$ were obtained and tested on INSTRON 1195 Testing Machine. Fatigue specimens with an unnotched hourglass shaped specimen with threaded grip sections and $6 \mathrm{~mm}$ in diameter (the minimum gage sections) and $76 \mathrm{~mm}$ in length were performed on ZwickAmsler HFP 5100 with triangular waveforms at room temperature under a load ratio of $\mathrm{R}=$ -1 .

Fractographic surface and cyclic deformation behavior after fatigue were observed using a field emission scanning electron microscope (FESEM) of HITACHI SU6600. The dislocation evolution was observed by transmission electron microscope (JEM200CX) operating at $120 \mathrm{KV}$. Based on the electronbackscattered diffraction (EBSD), using a field emission gun SEM (Carl Zeiss Microscopy GmbH 73447, ZEISS) equipped with an HKL EBSD system, the strain distribution on the fatigue initiation site was investigated. The microstructure parameters were obtained by the Image Pro-Plus 6.0 software.

\section{Results and discussions}

\subsection{Microstructure before fatigue}

Fig. 1 shows the microstructures before fatigue. The bimodal microstructure consists of three different $\alpha$ morphology, including the equiaxed primary $\alpha\left(\alpha_{\mathrm{p} 1}\right)$, the primary $\alpha$ laths $\left(\alpha_{\mathrm{p} 2}\right)$ and the secondary acicular $\alpha$ phase $\left(\alpha_{\mathrm{s}}\right)$ in the $\beta$ matrix ( $\left.\beta_{\text {trans }}\right)$. The microstructural parameters for $\mathrm{BM}$ are listed in table 2. The average width of $\alpha$ phase in FLM is about $180 \mathrm{~nm}$.

Table 2. Microstructure parameters for the BM

\begin{tabular}{|c|c|c|c|}
\hline Structures & Size & $\begin{array}{c}\text { Volume fraction } \\
(\%)\end{array}$ & Aspect \\
\hline$\alpha_{\mathrm{p} 1}$ & $10.1 \mu \mathrm{m}$ & 25.4 & 1.7 \\
\hline$\alpha_{\mathrm{p} 2}$ & $2.2 \mu \mathrm{m}$ & 13.1 & 6.3 \\
\hline$\alpha_{\mathrm{s}}$ & $100 \mathrm{~nm}$ & & \\
\hline$\beta_{\text {trans }}$ & & 61.5 & \\
\hline
\end{tabular}

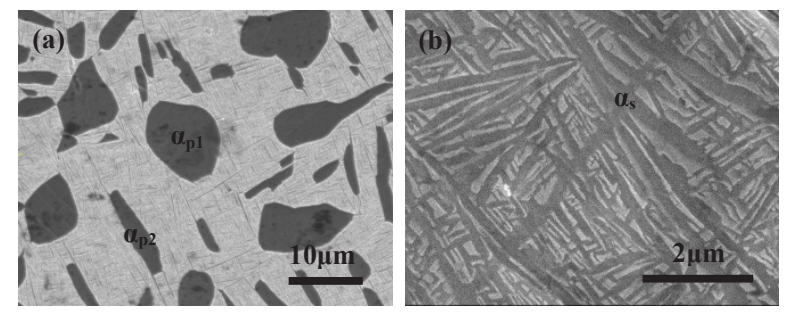

Fig. 1. Microstructures before fatigue: (a) BM; (b) FLM.

\subsection{Mechanical properties}

The mechanical properties for BM and FLM are listed in table 3. It can be seen that the FLM possesses lower ductility but higher yield and tensile strength than the BM one. The variations of the maximum stress with fatigue life for BM and FLM are compared. It indicates that FLM shows higher high cycle fatigue strength than $\mathrm{BM}$ at room temperature with a load ratio of -1 . The fatigue strength for BM and FLM at $10^{7}$ cycles with a stress ratio of minus one is approximately $490 \mathrm{MPa}$ and $650 \mathrm{MPa}$, respectively.

Table 3. Tensile results for the two microstructures

\begin{tabular}{|c|c|c|c|c|}
\hline Structures & $\sigma_{\mathrm{s}}(\mathrm{MPa})$ & $\begin{array}{c}\sigma_{\mathrm{b}} \\
(\mathrm{MPa})\end{array}$ & $\varepsilon(\%)$ & $\begin{array}{c}\text { Fatigue } \\
\text { strength (MPa) }\end{array}$ \\
\hline $\mathrm{BM}$ & 1110 & 1240 & 12 & 490 \\
\hline FLM & 1210 & 1280 & 2 & 650 \\
\hline
\end{tabular}

The aforementioned empirical descriptions of fatigue life are for the fully reversed fatigue loading with the mean stress of zero. The mean stress level of the imposed fatigue cycle is known to play an important role in influencing the fatigue behavior of engineering materials [9]. The effect of mean stress on fatigue can also be represented in terms of constant-life diagrams which are often used as a design consideration in engineering applications [9]. In most cases, the popular Goodman model is used for the constant life diagrams $[9,13,26]$. In general, the Goodman formula can be expressed as: 


$$
\sigma_{\mathrm{a}}=\sigma_{-1} *\left(1-\frac{\sigma_{\mathrm{m}}}{\sigma_{\mathrm{b}}}\right)
$$

Where $\sigma_{\mathrm{a}}$ is the stress amplitude, $\sigma_{-1}$ is the fatigue limit $(\mathrm{R}=-1)$ of the alloy, $\sigma_{\mathrm{m}}$ is the mean stress, and $\sigma_{\mathrm{b}}$ is the ultimate tensile strength.

Fig. 2 shows the Goodman diagrams of BM and LM which is constructed under $10^{7}$ cycles at room temperature. The corresponding Goodman equations for BM (Eq. (2)) and LM (Eq. (3)) are as following:

$$
\begin{aligned}
& \sigma_{\mathrm{a}}=490 *\left(1-\frac{\sigma_{\mathrm{m}}}{1200}\right) \\
& \sigma_{\mathrm{a}}=650 *\left(1-\frac{\sigma_{\mathrm{m}}}{1280}\right)
\end{aligned}
$$

It indicated from Fig. 2 that LM presented a better HCF strength than those of BM no matter what the load ratio is ( $\mathrm{R}$ is from -1 to 1$)$. This is consistent with the above high cycle fatigue results $(\mathrm{R}=-1)$ from table 3 .

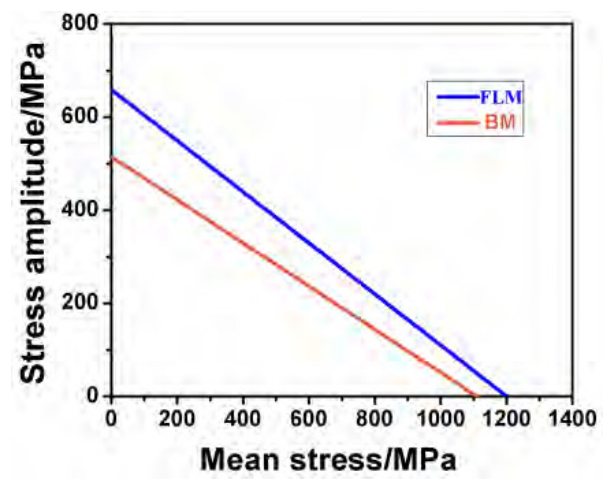

Fig. 2. Goodman diagrams of TC21 alloy with BM and FLM

\subsection{The crack initiation and coalescence behavior}

During the high cycle fatigue, most of the fatigue life is spent in the region of fatigue crack initiation. Therefore, it is essential to explore the relationship between fatigue crack nucleation and microstructures. The observation of second cracks and voids were performed near the site of the main crack, as described in details in Ref. [11]. Fig. 3 shows the fatigue crack initiation behavior of different microstructures. Most of the cracks nucleate at the primary $\alpha$ phase interface, including equiaxed and primary $\alpha$ phase in the BM at stress amplitude of 500 $\mathrm{MPa}$, as shown in Fig. 3a and b. Therein, cracks at the primary $\alpha / \beta_{\text {trans }}$ interface tend to connect with each other along the interface (Fig. 3b). However, fatigue cracks mainly initiate either at the intrusions or intersection of two intrusions with various orientations on the rough surface in FLM at stress amplitude of $690 \mathrm{MPa}$, as shown in Fig. 3c and d.
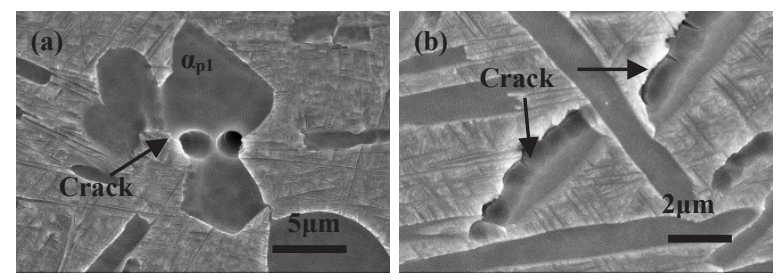
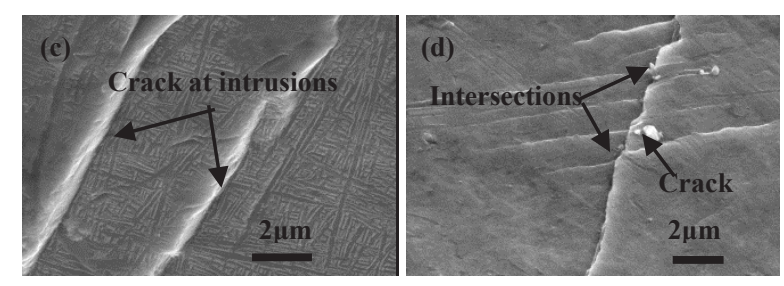

Fig. 3. Fatigue crack initiation behavior within different microstructures: (a-b) BM; and (c-d) FLM

The microcrack coalescence behavior is different from long cracks in titanium alloys [9, 11, 27-28]. Based on the observation of crack initiation behavior, it is found that crack easily nucleates at the primary $\alpha$ lath. Once initiated, it would connect with another crack to form large cracks, as shown in Fig. 4a. With further cycling, this crack propagates along the large $\alpha$ phase interface and finally transfers across the thin $\beta$ lath, as seen in Fig. 4b. It has been reported that serious local deformation existed at the primary $\alpha$ lath, which leads to stress concentration at the interface $[11,25,29]$.

However, fatigue crack mainly nucleates at the intrusions and propagates along the intrusions, as displayed in Fig. 4c. In most cases, these cracks are parallel to each other in the $\beta$ grain boundaries interior (Fig. 4d). Little crack is found at the $\beta$ grain boundaries in present work, which is different to the metastable titanium alloy $[13,15,27]$. Within the large $\beta$ grain, the only one chance to concatenate with each other is at the intersection site along two directions of extrusions, as shown in Fig. 4d. Its low capability for microcrack coalescence in FLM at the initial stage may improve the initiation fatigue life.
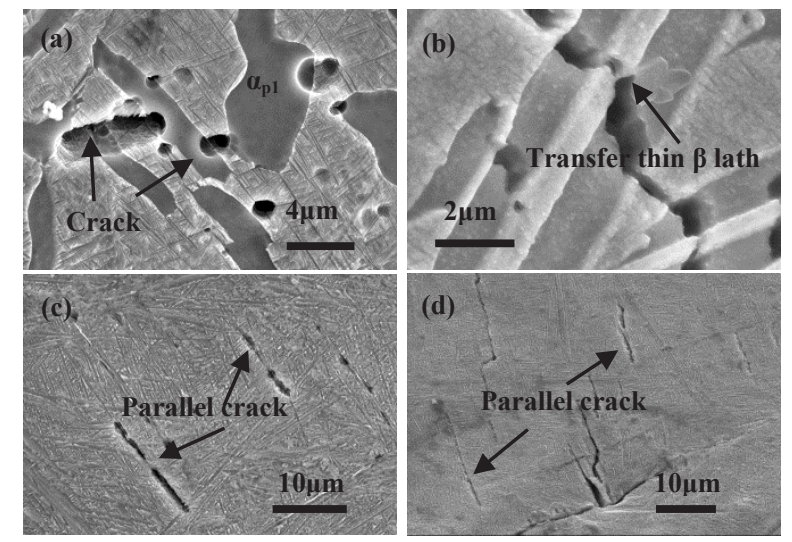

Fig. 4. Fatigue crack coalescence behavior within different microstructures: (a-b) BM; and (c-d) FLM

\subsection{The dislocation evolution}

Recently, Echlin et al [20] found that the localized plastic deformation was produced at some micro-regions in Ti-6Al-4V titanium alloys even at the elastic region. Additionally, it has been reported that the dislocation motion during each cyclic is not completely reversible and results in strain localization which leads to the crack formation [19, 22-24]. Thus, it is essential to investigate the dislocation structure during fatigue within various microstructures.

Fig. 5a-b illustrates various deformation processes for the primary $\alpha$ laths at stress amplitude of $560 \mathrm{MPa}$. 
Some (10 $\overline{10}$ ) primary slip bands occurred within $\alpha$ laths and interact with the interface (Fig. 5a), which leads to some slip steps and kinks. In addition to slip bands, some deformation twins have been activated to coordinate the plastic deformation in the primary $\alpha$ lath (Fig. 5b). For many metals, it has been reported that the main cyclic plastic deformation mechanism is dislocation motion, which is accomplished by slip or twinning in some cases [9-11,13,15]. Fig. 5c displays a number of $(10 \overline{1} 0)$ prismatic slip bands and dislocation arrays in the equiaxed primary $\alpha$ phase. Some dislocation debris and tangles exist in the $\beta_{\text {trans }}$ matrix, as Fig. $5 \mathrm{~d}$ shown.
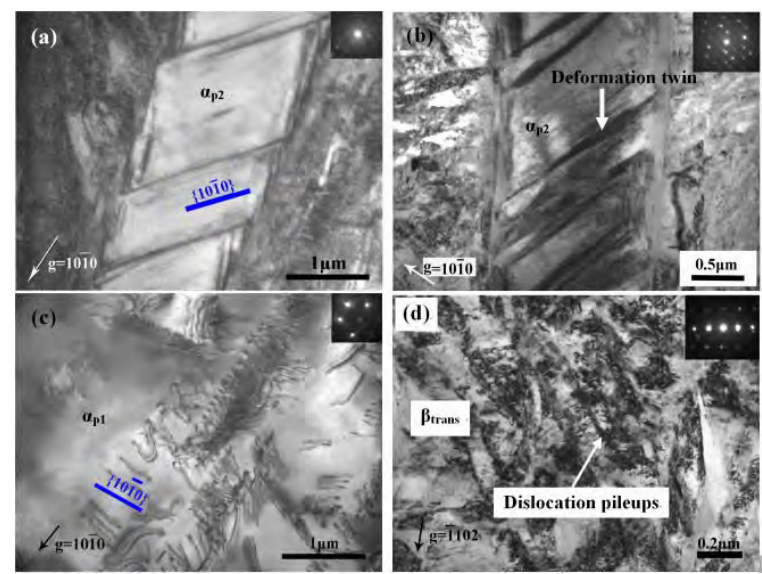

Fig. 5. The dislocation structures in BM at stress amplitude of $560 \mathrm{MPa}$ : (a) some prismatic slip bands in the primary $\alpha$ lath, (b) some deformation twins in primary $\alpha$ lath, (c) multi prismatic slip bands in equiaxed $\alpha$ phase, (d) dislocation pileups and tangles in the $\beta_{\text {trans }}$ matrix

Fig. 6 shows the dislocation structures at different stress amplitudes in the FLM. Besides the dislocation pileups at the interface, easy slip transmission across the $\alpha / \beta$ interface is observed at $660 \mathrm{MPa}$, as indicated by the arrows in Fig. 6a. Many researchers have found that high ability of slip transmission is an efficient way to release the serious stress concentration at the interface, and improves the fatigue crack resistance $[21,25,30]$. The dislocation produces both in $\alpha$ and $\beta$ phase (Fig. 6b), which indicates that a compatible deformation at the interface is induced [21, 25]. It has been reported that deformation twin would generate when the dislocation slip is not sufficient to coordinate plastic deformation $[10-11,13,15]$. Fig. 6c shows a lot of deformation twins in the secondary $\alpha$ phase with about $50 \mathrm{~nm}$ in widths at a high-stress amplitude of $705 \mathrm{MPa}$. Furthermore, abundant of secondary nano-twins with about $10 \mathrm{~nm}$ in widths are observed within the deformation twins, as shown in Fig. 6d. It has been reported that nanotwins promote the back and forth motion of dislocations during cyclic loading in $\mathrm{Cu}$ [31], which improves the fatigue life. A relatively homogeneous cyclic deformation generates within micro-regions in FLM due to the easy slip transmission and plentiful nano-twins.
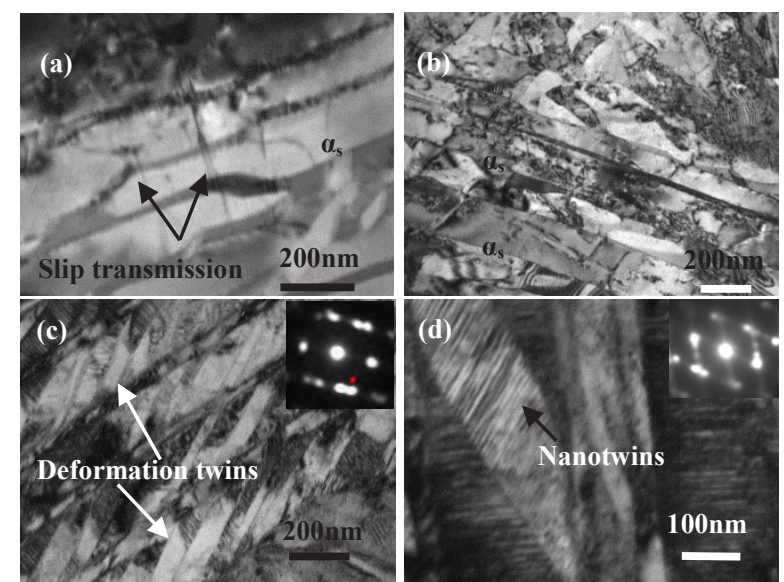

Fig. 6. The dislocation structures in FLM with different stress amplitude: (a) some slip transmission across the interface was observed at $660 \mathrm{MPa}$, (b) dislocation both in the $\alpha$ and $\beta$ phase was observed at $690 \mathrm{MPa}$, (c) some deformation twins were induced at $705 \mathrm{MPa}$, (d) abundant of nano-deformation twins at $705 \mathrm{MPa}$

\subsection{The non-uniform deformation}

To compare the heterogeneous cyclic deformation, especially at the fatigue crack initiation site, the microstructure and strain distribution after fatigue were examined by the EBSD. Fig. 7 shows the process to prepare the fatigue samples for the EBSD observation. Firstly, the fatigue crack initiation site was marked and then removed the rough surface, as shown in Fig. 7a. Secondary, the surface was metallographically prepared with $\mathrm{SiC}$ and electrolytic polished for the SEM and EBSD observation (Fig. 7b).
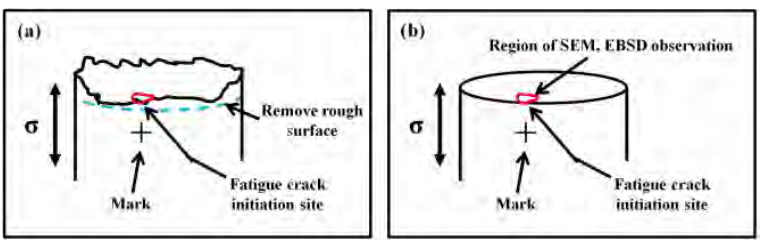

Fig. 7. The schematically illustration for SEM, EBSD observation near the fatigue crack initiation site: (a) a rough surface is removed, (b) regions of SEM, EBSD observation [10]

Fig. 8 shows the local misorientation and strain distribution at the fatigue crack initiation site both in BM and FLM. The large local misorientation tends to generate at the primary equiaxed $\alpha / \beta_{\text {trans }}$ interface, as shown in Fig. 8a. Fig. 8b indicates that the strain distribution is heterogeneous and easily localizes at the primary $\alpha$ lath. It has been reported that nonuniform deformation results in strain gradients and plastic interactions in the two-phase alloys [32-33]. Fatigue cracks tend to nucleate in this heterogeneous deformation region due to the high-stress concentration [11-13, 21, 33]. However, the local misorientation and strain distribution are relatively homogeneous in FLM than the BM one, as shown in Fig. 8c and d. Combined with the TEM results, it indicates that the cyclic deformation within micro-region is relatively homogeneous. Due to the uniform cyclic deformation and parallel cracks in FLM explain the better HCF strength than BM. 


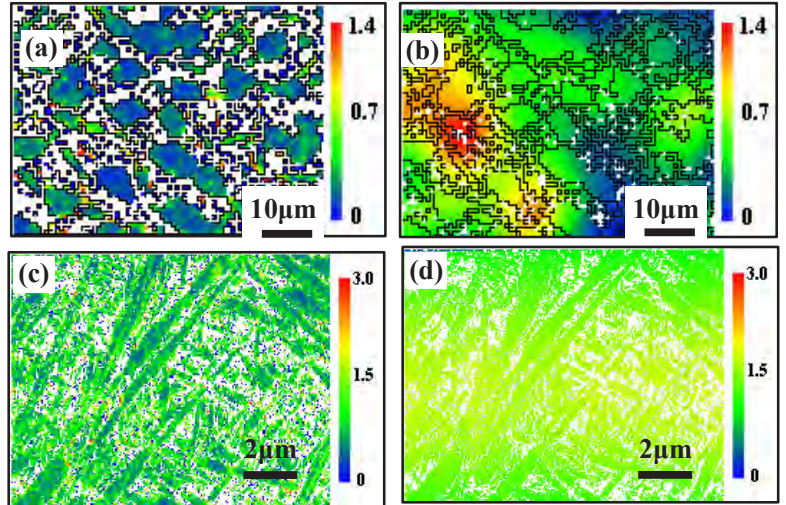

Fig. 8. The local misorientation and strain distribution near the fatigue crack initiation site within different microstructures: (ab) BM, (a) the local misorientation, (b) strain distribution; (c-d) FLM, (c) the local misorientation, (d) strain distribution

\section{Conclusions}

Based on the above experimental results and analysis, the following conclusions can be drawn:

(1) High cycle fatigue strength was obtained in FLM than the BM one.

(2) The corresponding Goodman equations for BM and FLM are as following:

BM : $\sigma_{\mathrm{a}}=490 *\left(1-\frac{\sigma_{\mathrm{m}}}{1200}\right) ;$

FLM: $\sigma_{\mathrm{a}}=650 *\left(1-\frac{\sigma_{\mathrm{m}}}{1280}\right)$.

(3) The heterogeneous plastic deformation existed within the large primary $\alpha$ phase. The cracks at interfaces and slip bands in the primary $\alpha$ lath easily coalesce with each other in BM. However, the $\alpha$ laths with similar morphology and size (nanosize) distributed uniformly in FLM. High ability of easy slip transmission across interface and abundant of nano-twins improves the fatigue initiation life.

(4) Based on the electron-backscattered diffraction (EBSD) analysis, it found that both the local misorientation and strain distribution were nonuniformly distributed in $\mathrm{BM}$, however, it is relatively homogeneous within micro-region in FLM, which delayed the initiation of the fatigue crack.

\section{Acknowledgments}

This project was financially supported by the 973 Program of China (2014CB644003), the National Natural Science Foundation of China (51671158, $51471129,51621063)$, and the 111 Project of China (B06025).

\section{References}

1. C. Leyens, M. Peters, Titanium and titanium alloys: fundamentals and applications, John Wiley \& Sons. (2003)
2. D. Banerjee, J.C. Williams. Acta Mater. 61(3) (2013)

3. G. Lütjering, J.C. Williams. Titanium, Berlin: Springer. (2003)

4. Y.Q. Zhao, G.J. Yang. Titanium Industry Progress. 23(5) (2006)

5. X.N. Wang, Z.S. Zhu, L. Tong, Y. Zhou, X.H. Zhou, H.Q. Yu. Rare Metals Letters. 27 (2008)

6. B.A. Cowles. Int. J. Fracture. 80(1996)

7. G.M. Castelluccio, W.D. Musinski, D.L. McDowell. Solid State Mater. Sci. 18 (2014).

8. A. Pineau, D.L. McDowell, E.P. Busso, S.D. Antolovich. Acta. Mater. 107 (2016)

9. S. Suresh. Fatigue of materials, Cambridge university press. (1998)

10. C.S. Tan, X.L. Li, Q.Y. Sun, L. Xiao, Y.Q. Zhao, J. Sun. Int. J. Fatigue. 75 (2015) 1-9

11. C.S. Tan, Q.Y. Sun, L. Xiao, Y.Q. Zhao, J. Sun. Mater. Sci. Eng. A. 711C (2018)

12. K.S. Chan. Int. J. Fatigue. 32(9) (2010)

13. C.W. Huang, Y.Q. Zhao, S.W. Xin, C.S. Tan, W. Zhou, Q. Li, W.D. Zeng. Int. J. Fatigue. 94 (2017)

14. C.W. Huang, Y.Q. Zhao, S.W. Xin, C.S. Tan, W. Zhou, Q. Li, W.D. Zeng. Mater. Sci. Eng. A. 682 (2017)

15. C.W. Huang, Y.Q. Zhao, S.W. Xin, W. Zhou, Q. Li, W.D. Zeng, C.S. Tan. J. Alloys Compd. 695 (2017)

16. G.Q. Wu, C.L. Shi, W. Sha, A.X. Sha, H.R. Jiang. Mater. Des. 46 (2013)

17. R.R. Boyer, J. Hall. Titanium'92: Sci Technol (1993)

18. P. Lukáš, M. Klesnil. Mater. Sci. Eng. A. 11(1973)

19. H. Mughrabi. Philos. Trans. R. Soc. London. Ser. A. 373(2038) (2015)

20. M.L.P. Echlin, J.C. Stinville, V.M. Miller, W.C. Lenthe, T.M. Pollock. Acta. Mater. 114 (2016)

21. W. Abuzaid, M.D. Sangid, J.D. Carroll, H. Sehitoglu, J. Lambros. J. Mech. Phys. Solids. 60(6) (2012)

22. H. Mughrabi. Acta Mater. 61(4) (2013)

23. H. Mughrabi. Metall. Mater. Trans. B. 40(4) (2009)

24. H. Mughrabi. Fatigue. Fract. Eng. Mater. Struct. 22(7) (1999)

25. C.S. Tan, Q.Y. Sun, L. Xiao, Y.Q. Zhao, J. Sun. J. Alloys. Compd. 724 (2017)

26. M.B. Bettaieb, A. Lenain, A.M. Habraken. Fatigue Fract. Eng. Mater. Struct. 36(5) (2013)

27. R.K. Nalla, B.L. Boyce, J.P. Campbell, J.O. Peters. Metall. Mater. Trans. A 33 (13)(2002)

28. S.G. Ivanova, R.R. Biederman, R.D. Sisson. J. Mater. Eng. Perform. 11 (2) (2002).

29. C.S. Tan, Q.Y. Sun, L. Xiao, Y.Q. Zhao, J. Sun. Mater. Sci. Eng. A. (Minor review)

30. L.L. Li, Z.J. Zhang, P. Zhang, J.B. Yang, Z.F. Zhang. Acta Mater. 120 (2016) 
31. Q.S. Pan, H.F. Zhou, Q.H. Lu, H.J. Gao, L. Lu. Nat. 551 (2017)

32. M.F. Ashby. Philos. Mag. 21(170) (1970)

33. S. Ankem, H. Margolin, C.A. Greene, B.W. Neuberger, P.G. Oberson. Prog. Mater. Sci. 51(5) (2006) 